\title{
Nucleophilic substitution and ring transformation reactions with 4-chloro-6-ethyl-3-nitropyrano[3,2-c]quinoline-2,5(6H)-dione
}

\author{
Hany M. Hassanin \\ Department of Chemistry, Faculty of Education, Ain Shams University, Roxy 11757, Cairo, \\ Egypt \\ E-mail: hanyhassnin@yahoo.com
}

\begin{abstract}
4-Chloro-6-ethyl-3-nitropyrano[3,2-c]quinoline-2,5(6H)-dione (3) was obtained by nitration followed by chlorination of 4-hydroxypyranoquinoline-2,5-dione 1. Substitution reactions of compound 3 with various nucleophiles, namely: sodium azide, amines, thiophenol and malononitrile, led to a series of novel 4-sustituted-3-nitropyranoquinolinones. Also, nucleophilic reactions of compound $\mathbf{3}$ with hydrazine, cyanoguanidine and $S$-methylisothiourea, involving ring opening-ring closure of the pyranoquinolinedione nucleus, are described.
\end{abstract}

Keywords: Pyrano[3,2-c]quinolines, nucleophilic substitution, pyrazolinone, furoxan, pyrimidine, ring opening-ring closure

\section{Introduction}

Pyrano[3,2-c]quinolinones are known as good synthons for many biologically important substituted quinolinones. Quinolin-2(1H)-one derivatives are of increasing interest since many of these compounds show useful applications as chemotherapeutic agents. For example, some quinolinones are recently reported to have potential as inhibitors for hepatitis C polymerase, ${ }^{1,2}$ microsomal prostaglandin $\mathrm{E}_{2}$ synthase- $1^{3}$ and selective $\mathrm{iNOS}^{4}$. Many quinolinone derivatives have been found active as antimalarial parasite agents, ${ }^{5}$ antiamebics, and antischistosomal agents, ${ }^{6-8}$ and to have antibacterial, ${ }^{9,10}$ antiproliferative and antitubulin, ${ }^{11}$ anti-hepatitis $B$ virus $(\mathrm{HBV})^{12,13}$ anti-HIV-1 ${ }^{14}$ activities.

Pyrano[3,2-c] quinolinones are able to undergo ring opening at C-2 and ring reclosure at C-4 when reacted with binucleophiles. ${ }^{15,16}$ The chemistry of nucleophilic reactions involving ring opening-ring closure (RORC) of 4-chloro-3-nitropyrano[3,2-c]quinolinone 3 attracted our attention due to the expected higher reactivity of the $o$-chloronitro heterocycles and to the paucity of their literature reports. ${ }^{17-19}$ On the other hand, the combination of a pyrazole and/or pyrimidine nucleus with the quinoline moiety in one molecular framework is reported to confer 
biological activity. ${ }^{20-22}$ Herein we report the synthesis of the novel 4-chloro-6-ethyl-3-nitropyrano[3,2-c] quinoline-2,5-dione (3) and a study of its chemical behavior towards some nitrogen, sulfur and carbon nucleophiles to obtain a new series of 4-substituted-3nitropyranoquinolinediones. Also, we use compound $\mathbf{3}$ to prepare 4-hydroxyquinolinones incorporating a pyrazolone or pyrimidine and/or triazolopyrimidine ring at position 3 , with the possibility to show biological activity.

\section{Results and Discussion}

Heating $N$-ethylaniline with two equivalents of diethyl malonate gave 4-hydroxypyrano[3,2-c]quinoline-2,5(6H)-dione (1). ${ }^{23,24}$ Nitration of compound $\mathbf{1}$ has been reported with a mixture of conc. nitric acid and conc. sulfuric acid in boiling acetic acid. ${ }^{25}$ We herein report for application of a known modification under substantial milder conditions, at room temperature using sodium nitrite as catalyst. ${ }^{17}$ Roschger et al. ${ }^{17}$ had applied this methodology in similar case of 4hydroxyquinolin-2-ones, and according to this modification, catalytic effect of the nitrite was attributed to an initial nitrosation at position 3 and subsequent in situ oxidation of the nitroso intermediate to the desired nitro product 2 (Scheme 1). Chlorination of compound 2 with phosphoryl chloride in the presence of triethylamine afforded 4-chloro-3-nitropyrano[3,2c] quinoline-2,5(6H)-dione 3 (Scheme 1). The IR and ${ }^{1} \mathrm{H}$ NMR spectra of compound 3 confirmed the absence of the hydroxy group. The mass spectrum of compound $\mathbf{3}$ showed two molecular ion peaks at $m / z 320\left[\mathrm{M}^{+}\right]$and $m / z 322\left[\mathrm{M}^{+}+2\right]$, these data support the identity of the structure and confirm the presence of the chlorine atom.

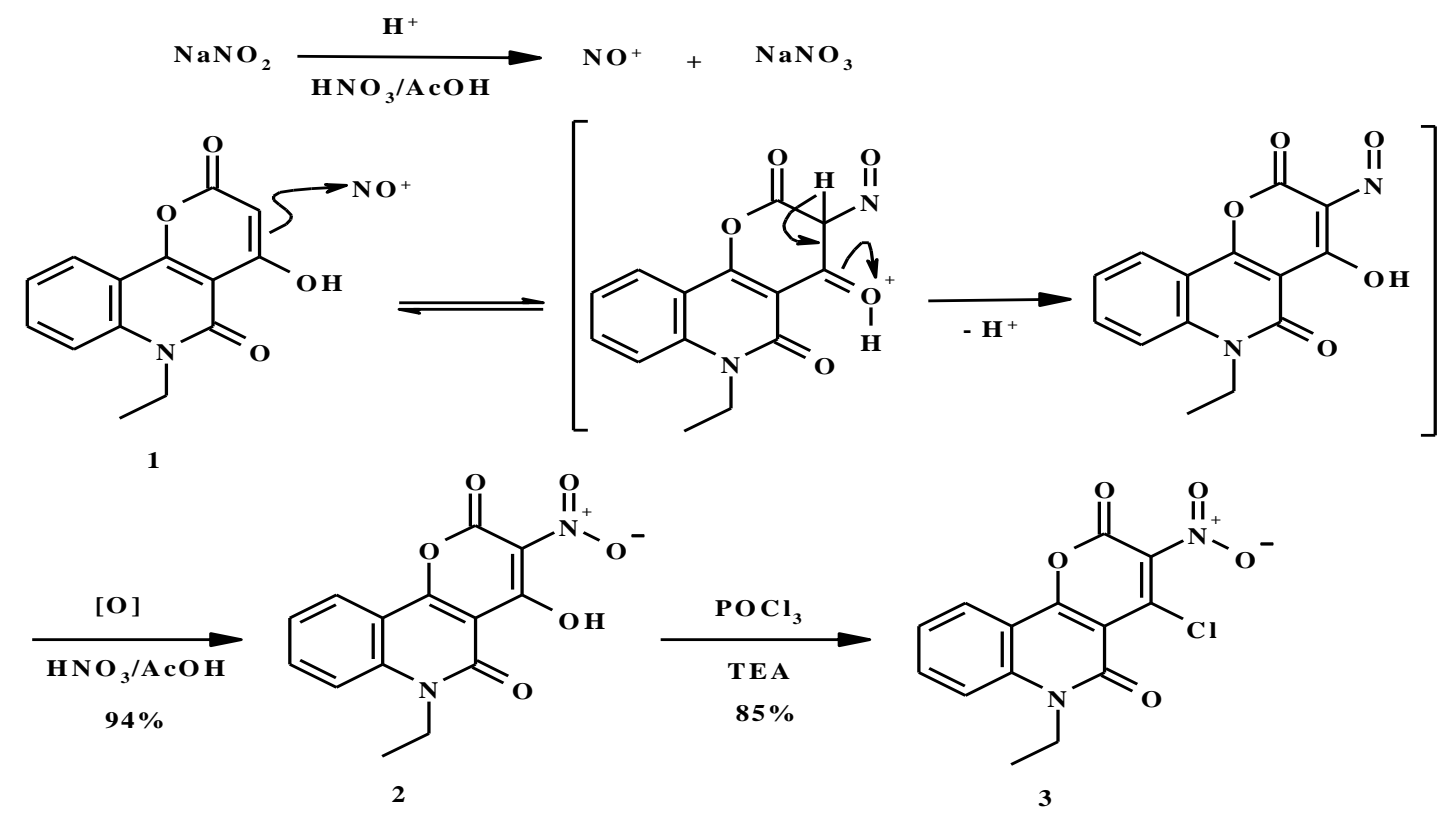

\section{Scheme 1}


The substitution of the chlorine by an azide group was achieved by the reaction of compound 3 with sodium azide, in $N$-methylpyrrolidone, at room temperature (Scheme 2). The IR spectrum of the product 4 showed characteristic absorption band at $2150 \mathrm{~cm}^{-1}$ attributed to the azido group. Organic azides with suitable ortho substituents are known to undergo thermal cyclization with loss of $\mathrm{N}_{2}$ gas. ${ }^{17,26,27}$ Ring closure to the fused furoxan $\mathbf{5}$ was achieved by thermolysis of the azide 4 in refluxing nitrobenzene (Scheme 2). The IR spectrum of furoxan $\mathbf{5}$ showed characteristic absorption bands at $1448 \mathrm{~cm}^{-1}$ assigned to the $\mathrm{C}=\mathrm{N}-\mathrm{O}$, while the stretching vibration of the fragment $\mathrm{O}-\mathrm{N} \rightarrow \mathrm{O}$ was seen at $1301 \mathrm{~cm}^{-1}$ (as the recently reported for furoxan ring $^{28}$ ). The mass spectrum of compound 5 showed the molecular ion peak at m/z 299 .

Nucleophilic substitution of the chlorine atom at position 4 of compound 3 by various nucleophiles such as amines, thiophenol, and malononitrile could be carried out under mild conditions. Thus, reaction of compound $\mathbf{3}$ with benzylamine led to the 4-benzylamino derivative 6a (Scheme 2). The ${ }^{1} \mathrm{H}$ NMR spectrum of compound $6 \mathbf{a}$ showed a new characteristic singlet signal at $\delta 4.26$ assigned to the methylene protons of benzyl group, in addition to one exchangeable signal at $\delta 10.00 \mathrm{ppm}$ due to NH proton. The reaction of compound 3 with aniline, in the presence of triethylamine, yielded the 4-phenylamino derivative $\mathbf{6 b}$ (Scheme 2). Notable in the ${ }^{1} \mathrm{H}$ NMR spectrum of compound $\mathbf{6 b}$ are the integral count of protons in the aromatic region revealing the presence of nine protons due to the aromatic protons of the quinoline and phenyl groups. In addition, the presence of a deuterium-exchangeable singlet appeared as a broad signal at $\delta 9.56$ due to N-H. Similarly, condensation of compound 3 with piperidine gave the 4-piperidinyl derivative 7 (Scheme 2). The ${ }^{1} \mathrm{H}$ NMR spectrum of compound 7 showed the signals due to the piperidinyl group as three characteristic multiplets at $\delta 1.33,1.62$ and 2.98 . The mass spectrum showed the molecular ion at $m / z, 369$, in agreement with the formula weight (369.38). Treatment of compound $\mathbf{3}$ with thiophenol, in the presence of triethylamine, afforded the thioether 8. In the ${ }^{13} \mathrm{C}$ NMR spectrum of $\mathbf{8}$ sixteen separate signals were observed at 95.8-160.9 ppm belonging to the aromatic carbon atoms, while the two carbonyls were seen at 170.6 and $170.9 \mathrm{ppm}$.

The introduction of a cyano group into compound $\mathbf{3}$ by Rosenmund-Braun aromatic cyanodechlorination with copper(I) cyanide in high-boiling solvents gave a mixture of compounds, ${ }^{29}$ in which separation attempts failed. However, another recently described method ${ }^{30}$ allowed us to introduce the carbonitrile function at the 4-position of compound $\mathbf{3}$ under relatively mild conditions. That is by a two-step reaction, the first step is converting $\mathrm{Cl}$ group to the reactive tosyloxy leaving group, at the 4-position, via the reaction of compound $\mathbf{3}$ with sodium $p$ toluenesulfonate to give 4-tosylate 9 (Scheme 3), which was then treated with potassium cyanide to afford the 4-cyanopyranoquinolinedione $\mathbf{1 0 .}$

The IR spectrum of compound $\mathbf{1 0}$ showed characteristic absorption bands at 2213, 1733, $1629 \mathrm{~cm}^{-1}$, attributed to $\mathrm{C} \equiv \mathrm{N}, \mathrm{C}=\mathrm{O}_{\text {pyrone }}$ and $\mathrm{C}=\mathrm{O}_{\text {quinolinone, }}$, respectively. Also, the mass spectrum showed the molecular ion peak at $\mathrm{m} / \mathrm{z} 311$ which is in good agreement with the formula weight (311.26). Reaction of compound $\mathbf{3}$ with malononitrile in ethanol containing few drops of triethylamine afforded 2-(pyranoquinolin-4-yl)malononitrile 11 (Scheme 3). The IR spectrum of 
compound 11 showed characteristic absorption bands at 2207, $2159(2 \mathrm{C} \equiv \mathrm{N}), 1737\left(\mathrm{C}=\mathrm{O}_{\text {pyrone }}\right)$ and $1623 \mathrm{~cm}^{-1}\left(\mathrm{C}=\mathrm{O}_{\text {quinolone }}\right)$. The malononitrile proton was observed at $\delta 5.57 \mathrm{ppm}$ in the ${ }^{1} \mathrm{H}$ NMR spectrum, while the $s p^{3}$ hybridized carbon atom of the malononitrile group appeared at $\delta$ $85.6 \mathrm{ppm}$ in the ${ }^{13} \mathrm{C}$ NMR spectrum.

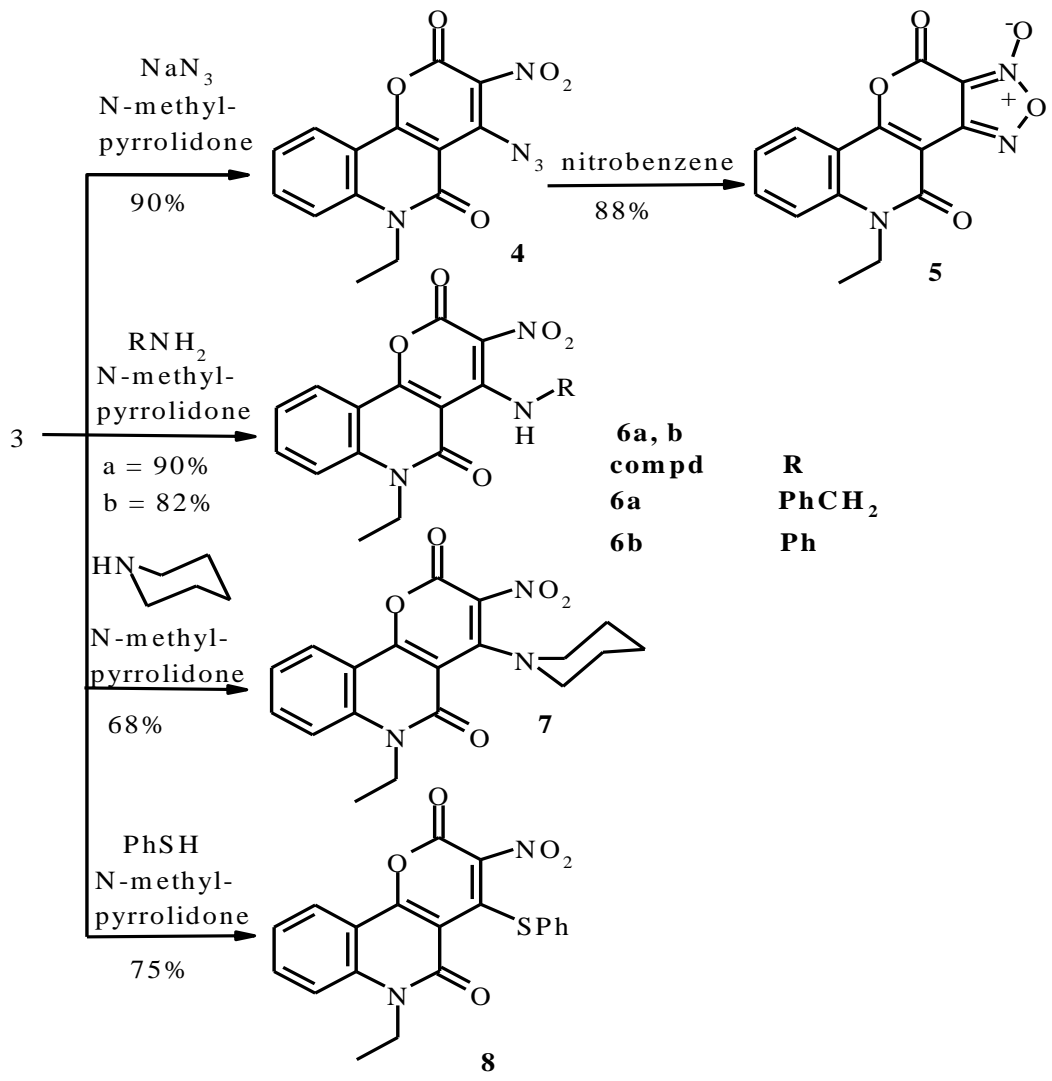

\section{Scheme 2}

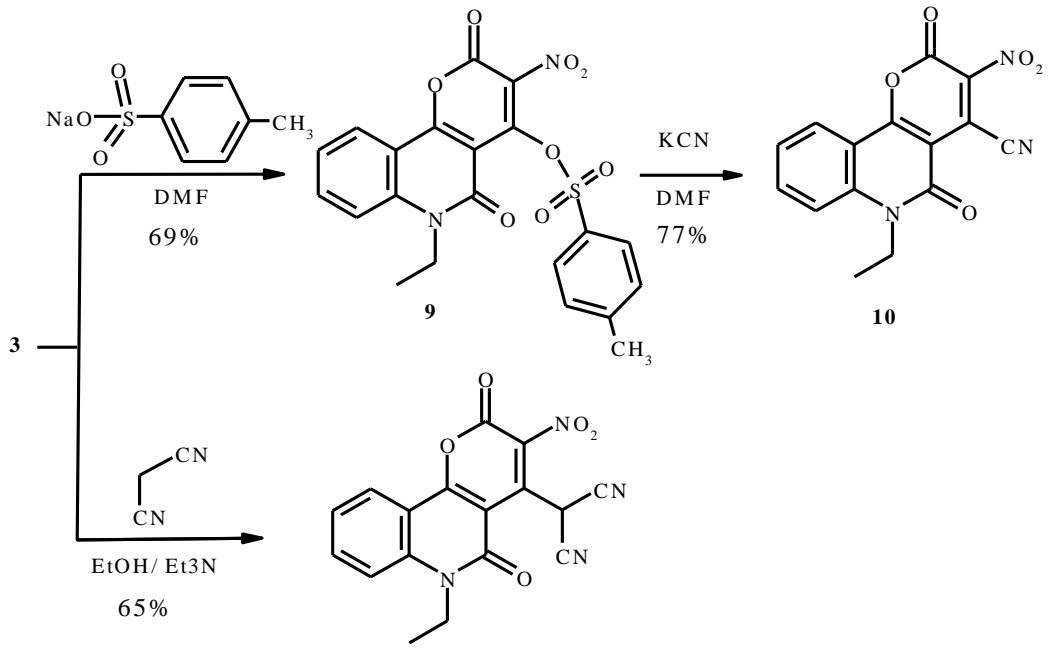

\section{Scheme 3}


The compound 3 was allowed to react with some binucleophilic reagents to prepare 4hydroxyquinolinones bearing a pyrazole or pyrimidine moiety in one molecular framework. Treatment of compound $\mathbf{3}$ with hydrazine hydrate in boiling DMF effected $\alpha$-pyrone ring opening followed by ring closure (RORC) with loss of $\mathrm{HCl}$, leading to the pyrazolone 12 (Scheme 4). The ${ }^{1} \mathrm{H}$ NMR spectrum of compound 12 showed signals due to three exchangeable protons characteristic for $2 \mathrm{NH}_{\text {pyrazole }}$ and $\mathrm{OH}_{\text {quinolinone }}$ at $\delta 11.30,13.41$ and $13.93 \mathrm{ppm}$. Also, the structure of compound 12 was supported by its mass spectrum which exhibited a molecular ion peak at $\mathrm{m} / \mathrm{z}$ 316. Reaction of compound $\mathbf{3}$ with cyanoguanidine as 1,3-binucleophile, afforded the pyrimidine derivative 13 (Scheme 4). The IR spectrum of pyrimidine 13 showed the presence of absorption bands at 3190 and $2199 \mathrm{~cm}^{-1}$, due to the $\mathrm{NH}$ and $\mathrm{C} \equiv \mathrm{N}$ groups, respectively. Furthermore, the ${ }^{1} \mathrm{H}$ NMR spectrum of compound $\mathbf{1 3}$ showed three deuterium-exchangeable singlet signals assignable to the two $\mathrm{NH}$ and the $\mathrm{OH}$ protons at $\delta 12.62,13.40$ and 13.95.

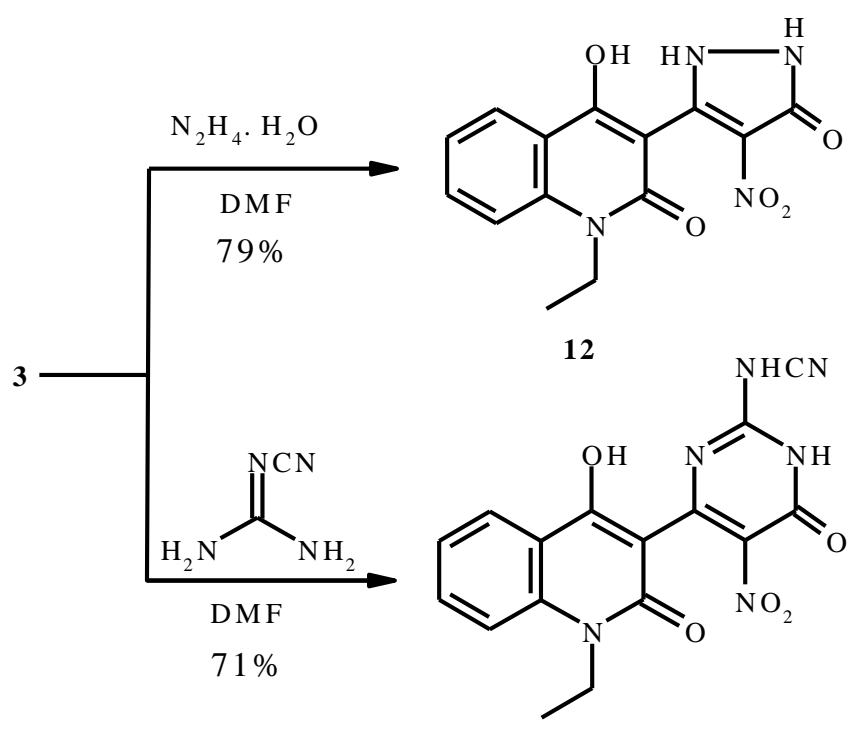

13

\section{Scheme 4}

The reaction of chloropyranoquinolinedione 3 with $S$-methylisothiourea in DMF afforded the methylsulfanylpyrimidine derivative 14 (Scheme 5). A methyl signal was observed at $\delta 2.83$ ppm in the ${ }^{1} \mathrm{H}$ NMR spectrum of $\mathbf{1 4}$, and at $\delta 22.1 \mathrm{ppm}$ in the ${ }^{13} \mathrm{C}$ NMR spectrum. The reaction of methylsulfanyl-pyrimidine derivative $\mathbf{1 4}$ with hydrazine hydrate in DMF produced the hydrazinopyrimidine 15 (Scheme 5). The elemental analysis showed absence of sulfur in the product revealing the replacement of the methylsulfanyl group. IR spectrum exhibited stretching vibrational bands at 3335,3191 and $3100 \mathrm{~cm}^{-1}$ due to $\mathrm{NH}_{2}$ and $\mathrm{NH}$ groups. ${ }^{1} \mathrm{H}$ NMR spectrum displayed two signals due to deuterium exchangeable protons at $\delta: 7.16$ and $8.25\left(-\mathrm{NH}-\mathrm{NH}_{2}\right)$. Moreover, the mass spectrum revealed a molecular ion peak at $m / z 358\left[\mathrm{M}^{+}\right]$as the base peak, in agreement with the calculated molecular weight of the product $\mathbf{1 5}$. Thermal cyclocondensation of the hydrazinopyrimidine 15 with triethyl orthoformate, in DMF, was carried out to get the triazolopyrimidine derivatives 16. The ${ }^{1} \mathrm{H}$ NMR spectrum of the cyclized product $\mathbf{1 6}$ revealed the 
disappearance of the $\mathrm{NH}_{2}$ signal which was appeared in the ${ }^{1} \mathrm{H}$ NMR spectrum of compound $\mathbf{1 5}$ at $\delta 7.16 \mathrm{ppm}$, in addition to the appearance of characteristic singlet signal at $\delta 8.22 \mathrm{ppm}$

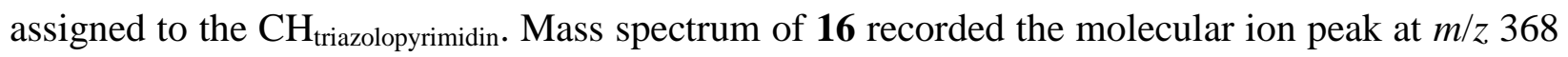
which agree well with the molecular formula and supports the identity of the structure.
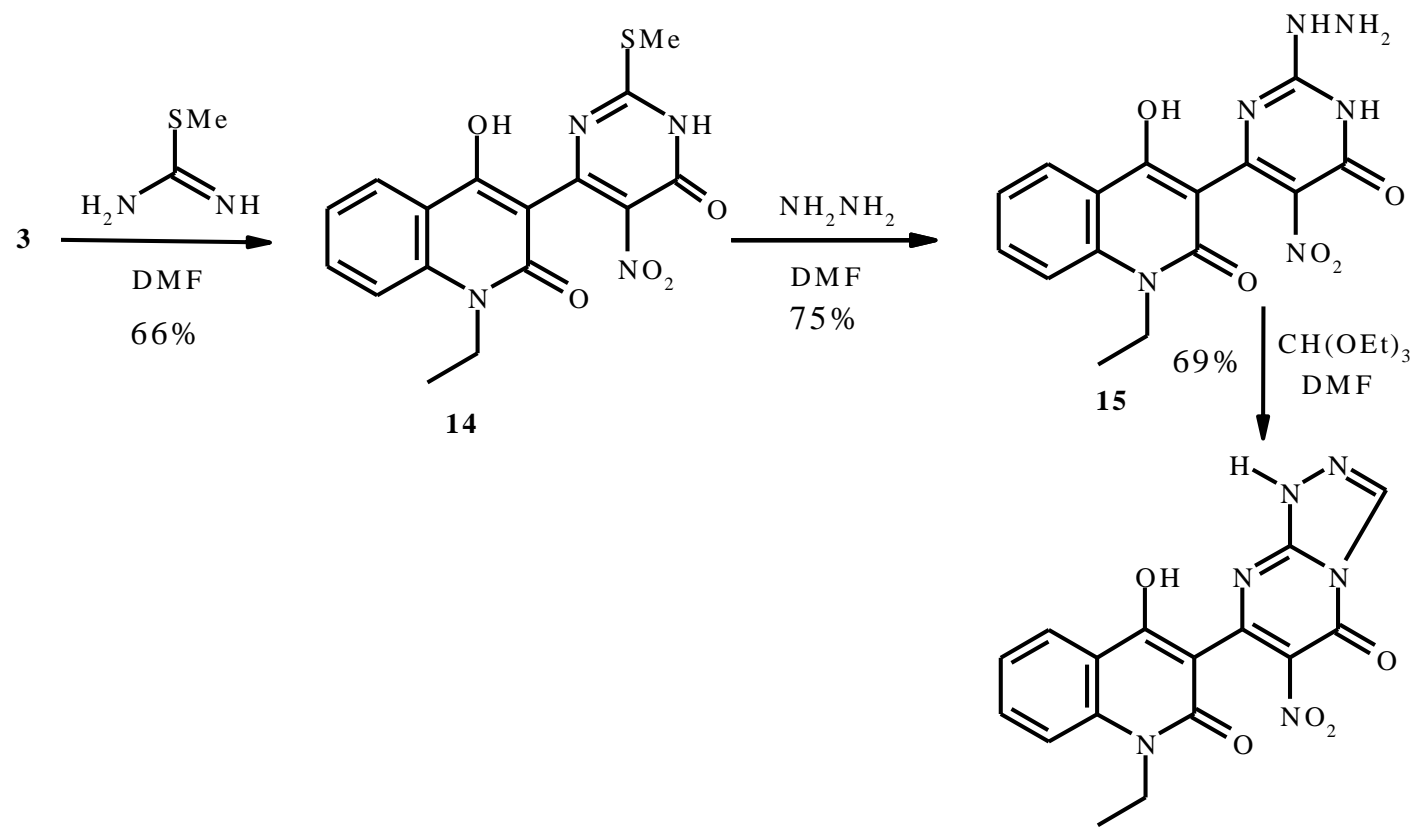

16

Scheme 5

\section{Conclusions}

4-Chloro-3-nitropyrano[3,2-c]quinoline-2,5(6H)-dione 3 was conveniently obtained via simple successful reactions. Many nucleophilic substitution reactions of the activated chloro leaving group in the compound $\mathbf{3}$ were carried out using facile and mild conditions. Combination of pyrazole and/or pyrimidine nuclei with quinolinone moiety in one molecular-frame was achieved via RORC heterocyclization reaction of compound $\mathbf{3}$ with some binucleophiles.

\section{Experimental Section}

General. Melting points were determined on a digital Stuart SMP3 apparatus. Infrared spectra were measured on Perkin-Elmer 293 spectrophotometer $\left(\mathrm{cm}^{-1}\right)$, using $\mathrm{KBr}$ disks. ${ }^{1} \mathrm{H}$ NMR spectra were measured on Gemini-300BB spectrometer $300 \mathrm{MHz}$ (at $75 \mathrm{MHz}$ for ${ }^{13} \mathrm{C}$ ), or Jeol Eca-500 MHz (at $125 \mathrm{MHz}$ for ${ }^{13} \mathrm{C}$ ) using DMSO- $d_{6}$ or $\mathrm{CDCl}_{3}$ as a solvent and TMS ( $\delta$ ) as the internal standard. Mass spectra were obtained using GC-2010 Shimadzu GC- Mass spectrometer $(70 \mathrm{eV})$. Elemental microanalyses were performed on a Perkin-Elmer CHN-2400 analyzer. 
6-Ethyl-4-hydroxy-3-nitropyrano[3,2-c]quinoline-2,5(6H)-dione (2). A suspension of compound 1 ( $2.57 \mathrm{~g}, 10 \mathrm{mmol})$ in glacial acetic acid $(20 \mathrm{~mL})$ was treated with concentrated nitric acid $(2 \mathrm{~mL}, 30 \mathrm{mmol})$ and sodium nitrite $(0.03 \mathrm{~g}, 0.5 \mathrm{mmol})$ to start slightly exothermic reaction. The starting material dissolved, followed immediately by precipitating the product. After stirring for 15 minutes the product was filtered, washed with water and crystallized from $\mathrm{DMF} / \mathrm{MeOH}$ to give compound 2 as yellow crystals, yield (2.85 g, 94\% ), m.p. 242-243 ${ }^{\circ} \mathrm{C}$ (the previously reported 90\%, 240-242).

4-Chloro-6-ethyl-3-nitropyrano[3,2-c]quinoline-2,5(6H)-dione (3). Dry triethylamine (1 mL) was added to a solution of compound $2(3.02 \mathrm{~g}, 10 \mathrm{mmol})$ in phosphoryl chloride $(15.2 \mathrm{~mL}, 100$ $\mathrm{mmol}$ ). The mixture was refluxed for $1 \mathrm{~h}$. The excess solvent was removed by distillation and the residue poured on ice water $(100 \mathrm{~mL})$. The precipitate so formed was filtered, washed with water, dried and crystallized from EtOH to give compound $\mathbf{3}$ as yellow crystals, yield (2.75 g, 85\%), m.p. 206-208 ${ }^{\circ} \mathrm{C}$. IR $\left(\mathrm{KBr}, \mathrm{cm}^{-1}\right)$ : $3081\left(\mathrm{CH}_{\text {arom. }}\right), 2975,2930\left(\mathrm{CH}_{\text {aliphatic }}\right), 1732$ $\left(\mathrm{C}=\mathrm{O}_{\text {pyranone }}\right), 1636\left(\mathrm{C}=\mathrm{O}_{\text {quinolinone }}\right), 1616(\mathrm{C}=\mathrm{C}), 1567,1371\left(\mathrm{NO}_{2}\right), 756(\mathrm{C}-\mathrm{Cl}) .{ }^{1} \mathrm{H}$ NMR $(500$ MHz, DMSO- $\left.d_{6}, \delta\right): 1.19\left(\mathrm{t}, 3 \mathrm{H}, J=6.9 \mathrm{~Hz}, \mathrm{CH}_{2} \mathrm{CH}_{3}\right), 4.34\left(\mathrm{q}, 2 \mathrm{H}, J=6.9 \mathrm{~Hz}, \mathrm{CH}_{2} \mathrm{CH}_{3}\right), 7.38$ $(\mathrm{t}, 1 \mathrm{H}, J=7.2 \mathrm{~Hz}, \mathrm{H}-9), 7.67$ (d, 1H, $J=8.0 \mathrm{~Hz}, \mathrm{H}-7), 7.82$ (t, 1H, J = 7.2 Hz, H-8), 8.11 (d, 1H, $J=8.0 \mathrm{~Hz}, \mathrm{H}-10) .{ }^{13} \mathrm{C}$ NMR $\left(125 \mathrm{MHz}, \mathrm{DMSO}-d_{6}, \delta\right): 13.1\left(\mathrm{CH}_{3}\right), 38.2\left(\mathrm{CH}_{2}\right), 99.3\left(\mathrm{C}_{4 \mathrm{a}}\right), 113.3$ $\left(\mathrm{C}_{7}\right), 117.0\left(\mathrm{C}_{10 \mathrm{a}}\right), 118.9\left(\mathrm{C}_{9}\right), 124.8\left(\mathrm{C}_{10}\right), 125.5\left(\mathrm{C}_{8}\right), 138.8\left(\mathrm{C}_{6 \mathrm{a}}\right), 153.3\left(\mathrm{C}_{4}\right), 159.4\left(\mathrm{C}_{10 \mathrm{~b}}\right.$ as $\mathrm{C}-$ $\mathrm{O}), 163.1\left(\mathrm{C}_{3}\right), 164.0\left(\mathrm{C}_{5}\right.$ as $\left.\mathrm{C}=\mathrm{O}\right), 166.4\left(\mathrm{C}_{2}\right.$ as $\left.\mathrm{C}=\mathrm{O}\right) . \mathrm{M} / \mathrm{z}$ (relative intensity): $322\left[\mathrm{M}^{+}+2,13\right]$, $321\left[\mathrm{M}^{+}+1,23\right], 320$ [M+, 48], 319 (41), 308 (13), 292 (12), 248 (16), 218 (21), 190 (33), 162 (16), 146 (100), 132 (47), 77 (61). Anal. Calcd for $\mathrm{C}_{14} \mathrm{H}_{9} \mathrm{~N}_{2} \mathrm{O}_{5} \mathrm{Cl}$ (320.69): C, 52.44; H, 2.83; N, 8.74; Cl, 11.06\%. Found: C, 52.20; H, 2.79; N, 8.23; Cl, $10.98 \%$.

4-Azido-6-ethyl-3-nitropyrano[3,2-c]quinoline-2,5(6H)-dione (4). A solution of compound 3 (3.20 g, $10 \mathrm{mmol})$ and sodium azide $(0.98 \mathrm{~g}, 15 \mathrm{mmol})$ in $N$-methylpyrrolidone $(30 \mathrm{~mL})$ was stirred at room temperature for $3 \mathrm{~h}$. then the reaction mixture was poured into $500 \mathrm{~mL}$ of ice water, the precipitate so formed was filtered, washed with water and dried and crystallized from methanol to give compound 4 as yellow crystals, yield $(2.94 \mathrm{~g}, 90 \%)$, m.p. $128{ }^{\circ} \mathrm{C}$, partial decomposition, resolidifies and melts again at $171-173^{\circ} \mathrm{C}$. IR $\left(\mathrm{KBr}, \mathrm{cm}^{-1}\right): 3081\left(\mathrm{CH}_{\text {arom. }}\right), 2978$, $2874\left(\mathrm{CH}_{\text {aliphatic }}\right), 2150\left(\mathrm{~N}_{3}\right), 1719\left(\mathrm{C}=\mathrm{O}_{\text {pyranone }}\right), 1632\left(\mathrm{C}=\mathrm{O}_{\text {quinolinone }}\right), 1610(\mathrm{C}=\mathrm{C}), 1565,1366$ $\left(\mathrm{NO}_{2}\right) .{ }^{1} \mathrm{H}$ NMR $\left(300 \mathrm{MHz}, \mathrm{DMSO}-d_{6}, \delta\right): 1.22\left(\mathrm{t}, 3 \mathrm{H}, J=6.9 \mathrm{~Hz}, \mathrm{CH}_{2} \mathrm{CH}_{3}\right), 4.28(\mathrm{q}, 2 \mathrm{H}, J=6.9$ $\left.\mathrm{Hz}, \mathrm{CH}_{2} \mathrm{CH}_{3}\right), 7.23$ (t, $\left.1 \mathrm{H}, J=7.6 \mathrm{~Hz}, \mathrm{H}-9\right), 7.63$ (d, $\left.1 \mathrm{H}, J=8.0 \mathrm{~Hz}, \mathrm{H}-7\right), 7.89$ (t, $1 \mathrm{H}, J=7.6$ $\mathrm{Hz}, \mathrm{H}-8), 8.17(\mathrm{~d}, 1 \mathrm{H}, J=8.0 \mathrm{~Hz}, \mathrm{H}-10) . \mathrm{M} / \mathrm{z}$ (relative intensity): $328\left[\mathrm{M}^{+}+1,5\right], 327\left[\mathrm{M}^{+}, 8\right]$, 308 (5), 306 (5), 297 (5), 256 (7), 213 (15), 191 (7), 169 (8), 149 (32), 133 (10), 129 (19), 69 (100). Anal. Calcd for $\mathrm{C}_{14} \mathrm{H}_{9} \mathrm{~N}_{5} \mathrm{O}_{5}$ (327.26): C, 51.38; H, 2.77; N, 21.40\%. Found: C, 51.14; H, $2.53 ; \mathrm{N}, 21.12 \%$. 
5-Ethyl-[1,2,5]oxadiazolo[3',4:4,5]pyrano[3,2-c]quinoline-4,11(5H)-dione 1-oxide (5). A solution of compound $4(3.27 \mathrm{~g}, 10 \mathrm{mmol})$ in nitrobenzene $(50 \mathrm{~mL})$ was refluxed until evolution of nitrogen gas had stopped (about 15 minutes). Then the solvent was removed by distillation and the residue poured on diethyl ether $(30 \mathrm{~mL})$. The product was filtered, washed with diethyl ether and crystallized from toluene to give compound $\mathbf{5}$ as colorless crystals, yield $(2.65 \mathrm{~g}, 88 \%)$, m.p. $179-181^{\circ} \mathrm{C}$. IR $\left(\mathrm{KBr}, \mathrm{cm}^{-1}\right): 3088\left(\mathrm{CH}_{\text {arom. }}\right), 2979,2933\left(\mathrm{CH}_{\text {aliphatic }}\right), 1735\left(\mathrm{C}=\mathrm{O}_{\text {pyranone }}\right)$, $1668\left(\mathrm{C}=\mathrm{O}_{\text {quinolinone }}\right), 1625(\mathrm{C}=\mathrm{N}), 1615(\mathrm{C}=\mathrm{C}), 1561,1448,1301,1002,820,731$ (assigned to furoxane ring). ${ }^{1} \mathrm{H}$ NMR (300 MHz, DMSO- $d_{6}, \delta$ ): 1.27 (t, $3 \mathrm{H}, J=6.4 \mathrm{~Hz}, \mathrm{CH}_{2} \mathrm{CH}_{3}$ ), 4.39 (q, $\left.2 \mathrm{H}, J=6.4 \mathrm{~Hz}, \mathrm{CH}_{2} \mathrm{CH}_{3}\right), 7.58(\mathrm{t}, 1 \mathrm{H}, J=7.2 \mathrm{~Hz}, \mathrm{H}-9), 7.91(\mathrm{~d}, 1 \mathrm{H}, J=8.4 \mathrm{~Hz}, \mathrm{H}-7), 7.92$ (t, $1 \mathrm{H}, J=7.2 \mathrm{~Hz}, \mathrm{H}-8), 8.18$ (d, $1 \mathrm{H}, J=8.4 \mathrm{~Hz}, \mathrm{H}-10) .{ }^{13} \mathrm{C}$ NMR $\left(125 \mathrm{MHz}, \mathrm{DMSO}-d_{6}, \delta\right): 12.1$ $\left(\mathrm{CH}_{3}\right), 38.2\left(\mathrm{CH}_{2}\right), 99.2,113.6,118.6,121.2,124.5,128.5,129.5,136.1,157.4,158.2,163.2$, 164.4. M/z (relative intensity): $300\left[\mathrm{M}^{+}+1,15\right], 299$ [M+, 77], 298 (20), 270 (11), 258 (19), 257 (100), 256 (12), 229 (35), 215 (19), 185 (22), 146 (22), 132 (47), 100 (33), 77 (48). Anal. Calcd for $\mathrm{C}_{14} \mathrm{H}_{9} \mathrm{~N}_{3} \mathrm{O}_{5}$ (299.24): C, 56.19; H, 3.03; N, 14.04\%. Found: C, 56.04; H, 2.93; N, $13.74 \%$.

4-Benzylamino-6-ethyl-3-nitropyrano[3,2-c]quinoline-2,5(6H)-dione (6a). A mixture of compound $3(3.20 \mathrm{~g}, 10 \mathrm{mmol})$ and benzylamine $(1.09 \mathrm{~mL}, 10 \mathrm{mmol})$ in $N$-methylpyrrolidone $(30 \mathrm{~mL})$ was stirred at room temperature for $4 \mathrm{~h}$. The product was precipitated with $100 \mathrm{~mL}$ of ice water, filtered, washed with water and crystallized from $\mathrm{AcOH}$ to give compound $\mathbf{6}$ as pale brown crystals, yield $\left(3.55\right.$ g, 90\%), m.p. $191-193^{\circ} \mathrm{C}$. IR $\left(\mathrm{KBr}, \mathrm{cm}^{-1}\right): 3081\left(\mathrm{CH}_{\text {arom. }}\right), 2975$, $2930\left(\mathrm{CH}_{\text {aliphatic }}\right), 1732\left(\mathrm{C}=\mathrm{O}_{\text {pyranone }}\right), 1636\left(\mathrm{C}=\mathrm{O}_{\text {quinolinone }}\right), 1616(\mathrm{C}=\mathrm{N}), 1559(\mathrm{C}=\mathrm{C}), 1567,1371$ $\left(\mathrm{NO}_{2}\right) .{ }^{1} \mathrm{H}$ NMR $\left(300 \mathrm{MHz}, \mathrm{DMSO}-d_{6}, \delta\right): 1.19\left(\mathrm{t}, 3 \mathrm{H}, J=7.2 \mathrm{~Hz}, \mathrm{CH}_{2} \mathrm{CH}_{3}\right), 4.26(\mathrm{~s}, 2 \mathrm{H}), 4.47$ (q, $2 \mathrm{H}, \mathrm{J}=7.2 \mathrm{~Hz}, \mathrm{CH}_{2} \mathrm{CH}_{3}$ ), $6.90-8.06$ (m, 9H, Ar-H), 10.00 (s, 1H, NH exchangeable with $\left.\mathrm{D}_{2} \mathrm{O}\right) . \mathrm{M} / \mathrm{z}$ (relative intensity): $391\left[\mathrm{M}^{+}, 40\right], 254$ (91), 240 (75), 229 (15), 201 (13), 161 (68), 146 (43), 144 (25), 120 (63), 117 (35), 104 (25), 92 (10), 78 (100). Anal. Calcd for $\mathrm{C}_{21} \mathrm{H}_{17} \mathrm{~N}_{3} \mathrm{O}_{5}$ (391.39): C, 64.45; H, 4.38; N, 10.74\%. Found: C, 64.44; H, 3.93; N, 10.58\%.

6-Ethyl-3-nitro-4-phenylaminopyrano[3,2-c]quinoline-2,5(6H)-dione (6b). A mixture of compound $3(3.20 \mathrm{~g}, 10 \mathrm{mmol})$ and aniline $(0.91 \mathrm{~mL}, 10 \mathrm{mmol})$, in $N$-methylpyrrolidone (50 $\mathrm{mL}$ ) containing few drops of triethylamine, was stirred at room temperature for $4 \mathrm{~h}$. The product was precipitated with ice water $(100 \mathrm{~mL})$, filtered, washed with water and crystallized from toluene to give compound $\mathbf{6 b}$ as pale yellow crystals, yield $(3.10 \mathrm{~g}, 82 \%)$, m.p. $202-204^{\circ} \mathrm{C}$. IR $\left(\mathrm{KBr}, \mathrm{cm}^{-1}\right): 3195(\mathrm{NH}), 3060\left(\mathrm{CH}_{\text {arom. }}\right), 2963,2868\left(\mathrm{CH}_{\text {aliphatic }}\right), 1725\left(\mathrm{C}=\mathrm{O}_{\text {pyranone }}\right), 1632$ $\left(\mathrm{C}=\mathrm{O}_{\text {quinolinone }}\right), 1592(\mathrm{C}=\mathrm{C}) . \mathrm{M} / \mathrm{z}$ (relative intensity): $377\left[\mathrm{M}^{+}, 57\right], 367$ [ $\left.\mathrm{M}^{+}-1,44\right], 348$ (41), 333 (55), 295 (55), 269 (89), 217 (47), 190 (55), 189 (100), 146 (52), 132 (94), 77 (66). ${ }^{1} \mathrm{H}$ NMR $\left(500 \mathrm{MHz}, \mathrm{DMSO}-d_{6}, \delta\right): 1.20\left(\mathrm{t}, 3 \mathrm{H}, J=6.8 \mathrm{~Hz}, \mathrm{CH}_{2} \mathrm{CH}_{3}\right), 4.33\left(\mathrm{q}, 2 \mathrm{H}, J=7.0 \mathrm{~Hz}, \mathrm{CH}_{2} \mathrm{CH}_{3}\right.$ ), $7.26-7.42(\mathrm{~m}, 6 \mathrm{H}, J=7.2 \mathrm{~Hz}, \mathrm{H}-9$ and Ar-H), $7.56(\mathrm{~d}, 1 \mathrm{H}, J=8.0 \mathrm{~Hz}, \mathrm{H}-7), 7.82(\mathrm{t}, 1 \mathrm{H}, J=$ $7.2 \mathrm{~Hz}, \mathrm{H}-8), 8.11$ (d, 1H, $J=8.0 \mathrm{~Hz}, \mathrm{H}-10), 9.56$ (s, 1H, NH exchangeable with $\mathrm{D}_{2} \mathrm{O}$ ). M/z (relative intensity): $377\left[\mathrm{M}^{+}, 57\right], 376\left[\mathrm{M}^{+}-1,45\right], 350$ (55), 333 (55), 332 (42), 310 (59), 295 (55), 288 (57), 269 (90), 255 (42), 241 (74), 217 (47), 189 (100), 174 (87), 161 (75), 146 (52), 
132 (94), 77 (67). Anal. Calcd for $\mathrm{C}_{20} \mathrm{H}_{15} \mathrm{~N}_{3} \mathrm{O}_{5}$ (377.36): C, 63.66; H, 4.01; N, 11.14\%. Found: C, 63.45; H, 3.83; N, $11.07 \%$.

6-Ethyl-3-nitro-4-piperidin-1-yl-pyrano[3,2-c]quinoline-2,5(6H)-dione (7). A mixture of compound $3(3.20 \mathrm{~g}, 10 \mathrm{mmol})$ and piperidine $(0.99 \mathrm{~mL}, 10 \mathrm{mmol})$, in $N$-methylpyrrolidone (30 $\mathrm{mL}$ ) was stirred at room temperature for $4 \mathrm{~h}$. The product was precipitated with ice water (100 $\mathrm{mL}$ ) and after standing $24 \mathrm{~h}$, filtered, washed with water and crystallized from toluene to give compound 7 as pale yellow crystals, yield $(2.51 \mathrm{~g}, 68 \%)$, m.p. $180-182^{\circ} \mathrm{C}$. IR $\left(\mathrm{KBr}, \mathrm{cm}^{-1}\right): 3092$ $\left(\mathrm{CH}_{\text {arom}}\right), 2985,2930$ ( $\left.\mathrm{CH}_{\text {aliphatic }}\right), 1733\left(\mathrm{C}=\mathrm{O}_{\text {pyranone }}\right), 1633\left(\mathrm{C}=\mathrm{O}_{\text {quinolinone }}\right), 1610(\mathrm{C}=\mathrm{N}), 1566$ $(\mathrm{C}=\mathrm{C}), 1565,1367\left(\mathrm{NO}_{2}\right) .{ }^{1} \mathrm{H}$ NMR $\left(300 \mathrm{MHz}, \mathrm{DMSO}-d_{6}, \delta\right): 1.19\left(\mathrm{t}, 3 \mathrm{H}, J=6.8 \mathrm{~Hz}, \mathrm{CH}_{2} \mathrm{CH}_{3}\right)$, $1.33(\mathrm{~m}, 2 \mathrm{H}), 1.62(\mathrm{~m}, 4 \mathrm{H}), 2.98(\mathrm{~m}, 4 \mathrm{H}), 4.24\left(\mathrm{q}, 2 \mathrm{H}, J=6.8 \mathrm{~Hz}, \mathrm{CH}_{2} \mathrm{CH}_{3}\right), 7.36(\mathrm{t}, 1 \mathrm{H}, J=7.4$ $\mathrm{Hz}, \mathrm{H}-9), 7.71$ (d, 1H, $J=8.0 \mathrm{~Hz}, \mathrm{H}-7), 7.98$ (t, 1H, $J=7.4 \mathrm{~Hz}, \mathrm{H}-8), 8.16$ (d, 1H, $J=8.0 \mathrm{~Hz}$, $\mathrm{H}-10) . \mathrm{M} / \mathrm{z}$ (relative intensity): $369\left[\mathrm{M}^{+}, 13\right], 368$ (4), 350 (12), 351 (11), 342 (13), 304 (11), 300 (11), 263 (10), 257 (4), 185 (12), 149 (22), 141 (49), 139 (100), 111 (60), 98 (22), 85 (10), 75 (23). Anal. Calcd for $\mathrm{C}_{19} \mathrm{H}_{19} \mathrm{~N}_{3} \mathrm{O}_{5}$ (369.38): C, 61.78; H, 5.18; N, 11.38\%. Found: C, 61.64; H, $5.03 ; \mathrm{N}, 11.09 \%$.

6-Ethyl-3-nitro-4-phenylsulfanylpyrano[3,2-c] quinoline-2,5(6H)-dione (8). A mixture of compound 3 (3.20 g, $10 \mathrm{mmol})$, thiophenol $(1.05 \mathrm{~mL}, 10 \mathrm{mmol})$ and few drops of triethylamine in $N$-methylpyrrolidone $(30 \mathrm{~mL})$ was stirred at room temperature for $1 \mathrm{~h}$. The product was precipitated with ice water $(100 \mathrm{~mL})$, filtered, washed with water and crystallized from toluene to give compound 8 as pale brown crystals, yield $(2.95 \mathrm{~g}, 75 \%)$, m.p. $222-224^{\circ} \mathrm{C}$. IR $\left(\mathrm{KBr}, \mathrm{cm}^{-}\right.$ $\left.{ }^{1}\right)$ : $3084\left(\mathrm{CH}_{\text {arom. }}\right), 2974,2937$ ( $\left.\mathrm{CH}_{\text {aliphatic }}\right), 1731$ (C=O $\left.\mathrm{O}_{\text {pyranone }}\right), 1670\left(\mathrm{C}=\mathrm{O}_{\text {quinolinone }}\right), 1613(\mathrm{C}=\mathrm{C})$, 1569, $1376\left(\mathrm{NO}_{2}\right) .{ }^{1} \mathrm{H}$ NMR (500 MHz, $\left.\mathrm{CDCl}_{3}, \delta\right): 1.24\left(\mathrm{t}, 3 \mathrm{H}, J=6.0 \mathrm{~Hz}, \mathrm{CH}_{2} \mathrm{CH}_{3}\right), 4.41$ (q, $\left.2 \mathrm{H}, J=7.2 \mathrm{~Hz}, \mathrm{CH}_{2} \mathrm{CH}_{3}\right), 7.25-8.23(\mathrm{~m}, 9 \mathrm{H}, \mathrm{Ar}-\mathrm{H}) .{ }^{13} \mathrm{C}$ NMR $\left(125 \mathrm{MHz}, \mathrm{DMSO}-d_{6}, \delta\right): 12.7$ $\left(\mathrm{CH}_{3}\right), 36.8\left(\mathrm{CH}_{2}\right), 95.8\left(\mathrm{C}_{4 \mathrm{a}}\right), 112.2,112.4,114.9,115.1,119.1,122.2,122.4,124.6,128.6$, 128.8, 134.5, 138.6, $148.0\left(\mathrm{C}_{4}\right), 154.1\left(\mathrm{C}_{10 \mathrm{~b}}\right.$ as $\left.\mathrm{C}-\mathrm{O}\right), 160.9\left(\mathrm{C}_{3}\right), 170.6\left(\mathrm{C}_{5}\right.$ as $\left.\mathrm{C}=\mathrm{O}\right), 170.9\left(\mathrm{C}_{2}\right.$ as $\mathrm{C}=\mathrm{O}) . \mathrm{M} / \mathrm{z}$ (relative intensity): 394 [M+1 12], 393 (10), 323 (23), 322 (100), 321 (60), 294 (65), 286 (38), 268 (46), 237 (20), 221 (19), 169 (13), 161 (16), 146 (23), 132 (34), 130 (20), 120 (32), 119 (27), 108 (25), 77(42). Anal. Calcd for $\mathrm{C}_{20} \mathrm{H}_{14} \mathrm{~N}_{2} \mathrm{O}_{5} \mathrm{~S}$ (394.41): C, 60.91; H, 3.58; N, 7.10; S, $8.13 \%$. Found: C, 60.70; H, 3.51; N, 7.04\%.

4-Methyl(6-ethyl-3-nitro-2,5-dioxo-5,6-dihydro-2H-pyrano[3,2-c]quinolin-4-yl) benzenesulfonate (9). A mixture of compound $3(3.20 \mathrm{~g}, 10 \mathrm{mmol})$ and sodium $p$-toluenesulfonate (1.94 $\mathrm{g}, 10 \mathrm{mmol})$ in DMF $(50 \mathrm{~mL})$ was heated at $120{ }^{\circ} \mathrm{C}$ for $20 \mathrm{~h}$. The product was precipitated with ice water $(100 \mathrm{~mL})$, filtered, washed with water and crystallized from ethanol to give $\mathbf{9}$ as pale yellow crystals, yield $(3.14,69 \%)$, m.p. $292-294^{\circ} \mathrm{C}$. IR $\left(\mathrm{KBr}, \mathrm{cm}^{-1}\right): 3084\left(\mathrm{CH}_{\text {arom. }}\right), 2973,2872$ ( $\left.\mathrm{CH}_{\text {aliphatic }}\right), 1723\left(\mathrm{C}=\mathrm{O}_{\text {pyranone }}\right), 1632\left(\mathrm{C}=\mathrm{O}_{\text {quinolinone }}\right), 1613(\mathrm{C}=\mathrm{C}), 1566,1372\left(\mathrm{NO}_{2}\right) .{ }^{1} \mathrm{H}$ NMR $\left(300 \mathrm{MHz}, \mathrm{DMSO}-d_{6}, \delta\right): 1.20\left(\mathrm{t}, 3 \mathrm{H}, J=6.9 \mathrm{~Hz}, \mathrm{CH}_{2} \mathrm{CH}_{3}\right), 2.43$ (s, 3H, $\left.\mathrm{CH}_{3}\right), 4.33$ (q, $2 \mathrm{H}, J=$ $\left.6.9 \mathrm{~Hz}, \mathrm{CH}_{2} \mathrm{CH}_{3}\right), 7.34-7.47(\mathrm{~m}, 3 \mathrm{H}, \mathrm{H}-9$ and $\mathrm{Ar}-\mathrm{H}), 7.56-7.67(\mathrm{~m}, 3 \mathrm{H}, \mathrm{H}-7$ and Ar-H), 7.83 $(\mathrm{t}, 1 \mathrm{H}, J=7.2 \mathrm{~Hz}, \mathrm{H}-8), 8.09$ (d, $1 \mathrm{H}, J=8.0 \mathrm{~Hz}, \mathrm{H}-10) .{ }^{13} \mathrm{C}$ NMR $\left(125 \mathrm{MHz}, \mathrm{DMSO}-d_{6}, \delta\right): 16.1$ $\left(\mathrm{CH}_{3}\right), 20.3\left(\mathrm{PhCH}_{3}\right), 38.2\left(\mathrm{CH}_{2}\right), 92.3,112.2,113.6,114.4,115.7,117.1,119.7,127.3,128.2$, $129.2,130.5,134.6,138.5,146.2,146.4,152.3,152.5,157.3 . \mathrm{M} / \mathrm{z}$ (relative intensity): $457\left[\mathrm{M}^{+}\right.$ 
+1, 13], 456 [M+ 44], 441 (11), 428 (14), 310 (37), 281 (41), 258 (11), 257 (51), 229 (100), 228 (53), 201 (43), 173 (26), 145 (46), 120 (22), 104 (26), 77 (48). Anal. Calcd for $\mathrm{C}_{21} \mathrm{H}_{16} \mathrm{~N}_{2} \mathrm{O}_{8} \mathrm{~S}$ (456.43): C, 55.26; H, 3.53; N, 6.14; S, 7.02\%. Found: C, 55.94; H, 3.54; N, 6.16; S, 7.05\%.

6-Ethyl-3-nitro-2,5-dioxo-5,6-dihydro-2H-pyrano[3,2-c]quinoline-4-carbonitrile (10). A mixture of compound $9(4.56 \mathrm{~g}, 10 \mathrm{mmol})$ and potassium cyanide $(0.79 \mathrm{~g}, 12 \mathrm{mmol})$ in dry DMF $(60 \mathrm{~mL})$ was stirred at $70{ }^{\circ} \mathrm{C}$ vigorously for $4 \mathrm{~h}$. Then, the mixture was poured into ice/water $(200 \mathrm{~mL})$ and . The obtained solid was filtered by suction, washed with water, dried and crystallized from dioxane to give compound $\mathbf{1 0}$ as yellow crystals, yield (2.4 g, 77\%), m.p. 286$288^{\circ} \mathrm{C}$. IR ( $\left.\mathrm{KBr}, \mathrm{cm}^{-1}\right)$ : $3080\left(\mathrm{CH}_{\text {arom. }}\right), 2924,2855\left(\mathrm{CH}_{\text {aliphatic }}\right), 2213(\mathrm{CN}), 1733\left(\mathrm{C}=\mathrm{O}_{\text {pyrane }}\right)$, $1629\left(\mathrm{C}=\mathrm{O}_{\text {quinolinone }}\right), 1611(\mathrm{C}=\mathrm{C}) .{ }^{1} \mathrm{H}$ NMR $\left(300 \mathrm{MHz}, \mathrm{DMSO}-d_{6}, \delta\right): 1.20(\mathrm{t}, 3 \mathrm{H}, J=6.8 \mathrm{~Hz}$, $\left.\mathrm{CH}_{2} \mathrm{CH}_{3}\right), 4.35$ (q, 2H, J=6.8 Hz, $\left.\mathrm{CH}_{2} \mathrm{CH}_{3}\right), 7.55$ (t, $\left.1 \mathrm{H}, J=7.2 \mathrm{~Hz}, \mathrm{H}-9\right), 7.91(\mathrm{~d}, 1 \mathrm{H}, J=8.0$ $\mathrm{Hz}, \mathrm{H}-7), 7.95$ (t, 1H, J=7.2 Hz, H-8), 8.14 (d, 1H, J=8.0 Hz, H-10). M/z (relative intensity): $312\left[\mathrm{M}^{+}+1,7\right], 311\left[\mathrm{M}^{+}, 26\right], 298$ (22), 297 (88), 285 (29), 268 (35), 239 (13), 191 (21), 190 (22), 161 (24), 146 (40), 133 (66), 130 (28), 119 (44), 104 (30), 77(100), 63(75). Anal. Calcd for $\mathrm{C}_{15} \mathrm{H}_{9} \mathrm{~N}_{3} \mathrm{O}_{5}$ (311.26): C, 57.88; H, 2.91; N, 13.50\%. Found: C, 57.64; H, 2.80; N, $12.74 \%$.

2-(6-Ethyl-3-nitro-2,5-dioxo-5,6-dihydro-2H-pyrano[3,2-c]quinolin-4-yl)-malononitrile (11). A mixture of compound $3(3.20 \mathrm{~g}, 10 \mathrm{mmol})$ and malononitrile $(0.70 \mathrm{~g}, 10 \mathrm{mmol})$, in absolute ethanol $(50 \mathrm{~mL})$ containing few drops of triethylamine, was stirred for $2 \mathrm{~h}$. The solid deposited after cooling was filtered and crystallized from methanol to give compound $\mathbf{1 1}$ as yellow crystals, yield $\left(2.27\right.$ g, 65\%), m.p. $216-218^{\circ} \mathrm{C}$. IR $\left(\mathrm{KBr}, \mathrm{cm}^{-1}\right): 3048\left(\mathrm{CH}_{\text {arom }}\right), 2974,2935,2852$ $\left(\mathrm{CH}_{\text {aliphatic }}\right), 2207,2159(2 \mathrm{CN}), 1737\left(\mathrm{C}=\mathrm{O}_{\text {pyranone }}\right), 1623(\mathrm{C}=\mathrm{O}), 1570(\mathrm{C}=\mathrm{C}) .{ }^{1} \mathrm{H}$ NMR $(300$ MHz, DMSO- $\left.d_{6}, \delta\right): 1.26\left(\mathrm{t}, 3 \mathrm{H}, J=7.8 \mathrm{~Hz}, \mathrm{CH}_{2} \mathrm{CH}_{3}\right), 4.38\left(\mathrm{q}, 2 \mathrm{H}, J=7.8 \mathrm{~Hz}, \mathrm{CH}_{2} \mathrm{CH}_{3}\right), 5.57$ $\left(\mathrm{s}, 1 \mathrm{H}, \mathrm{CH}(\mathrm{CN})_{2}\right), 7.52(\mathrm{t}, 1 \mathrm{H}, J=7.2 \mathrm{~Hz}, \mathrm{H}-9), 7.84-7.89(\mathrm{~m}, 2 \mathrm{H}, \mathrm{H}-7$ and H-8), $8.12(\mathrm{~d}, 1 \mathrm{H}, J$ $=8.0 \mathrm{~Hz}, \mathrm{H}-10) .{ }^{13} \mathrm{C} \mathrm{NMR}\left(75 \mathrm{MHz}, \mathrm{DMSO}-d_{6}, \delta\right): 12.7\left(\mathrm{CH}_{3}\right), 37.8\left(\mathrm{CH}_{2}\right), 85.6\left(\mathrm{CH}(\mathrm{CN})_{2}\right)$, $99.5\left(\mathrm{C}_{4 \mathrm{a}}\right), 113.0,116.1,116.3,123.9,124.3,124.6,134.6,134.8,137.5,157.1,157.5,162.0$, 165.5. M/z (relative intensity): 350 [ $\left.\mathrm{M}^{+}, 3\right], 349$ (2), 330 (4), 326 (4), 301 (27), 300 (79), 286 (6), 285 (16), 284 (20), 271 (27), 257 (57), 265 (45), 243 (39), 230 (40), 229 (100), 228 (48), 200 (44), 187 (35), 173(40), 144 (33), 116 (40), 77(48), 64 (58). Anal. Calcd for $\mathrm{C}_{17} \mathrm{H}_{10} \mathrm{~N}_{4} \mathrm{O}_{5}$ (350.29): C, 58.29; H, 2.88; N, 15.99\%. Found: C, 58.14; H, 2.83; N, $15.74 \%$.

\section{1-Ethyl-4-hydroxy-3-(4-nitro-5-oxo-2,5-dihydro-1H-pyrazol-3-yl)-quinolin-2(1H)-one (12).}

A mixture of compound $3(3.20 \mathrm{~g}, 10 \mathrm{mmol})$ and hydrazine hydrate $(0.58 \mathrm{~mL}, 12 \mathrm{mmol})$, in DMF (30 mL), was heated under reflux for $4 \mathrm{~h}$. The solid deposited after cooling was filtered and crystallized from acetic acid to give compound 12 as colorless crystals, yield (2.49 g, 79\%), m.p. 290-292 ${ }^{\circ}$ C. IR ( $\left.\mathrm{KBr}, \mathrm{cm}^{-1}\right): 3366(\mathrm{OH}), 3206(\mathrm{NH}), 3030\left(\mathrm{CH}_{\text {arom. }}\right), 2917\left(\mathrm{CH}_{\text {aliphatic }}\right), 1668$ $\left(\mathrm{C}=\mathrm{O}_{\text {pyrazol }}\right), 1632\left(\mathrm{C}=\mathrm{O}_{\text {quinolinone }}\right), 1586(\mathrm{C}=\mathrm{C}) .{ }^{1} \mathrm{H}$ NMR $\left(300 \mathrm{MHz}, \mathrm{DMSO}-d_{6}, \delta\right): 1.23(\mathrm{t}, 3 \mathrm{H}, J$ $\left.=7.4 \mathrm{~Hz}, \mathrm{CH}_{2} \mathrm{CH}_{3}\right), 4.32\left(\mathrm{q}, 2 \mathrm{H}, J=7.4 \mathrm{~Hz}, \mathrm{CH}_{2} \mathrm{CH}_{3}\right), 7.33$ (t, $\left.1 \mathrm{H}, J=7.2 \mathrm{~Hz}, \mathrm{H}-6\right), 7.59$ (d, 1H, $J=8.0 \mathrm{~Hz}, \mathrm{H}-8), 7.93$ (t, 1H, $J=7.2 \mathrm{~Hz}, \mathrm{H}-7), 8.07$ (d, 1H, $J=8.0 \mathrm{~Hz}, \mathrm{H}-5), 11.30,13.41$ (2s, $2 \mathrm{H}, 2 \mathrm{NH}$ exchangeable with $\left.\mathrm{D}_{2} \mathrm{O}\right), 13.93\left(\mathrm{~s}, 1 \mathrm{H}, \mathrm{OH}\right.$ exchangeable with $\left.\mathrm{D}_{2} \mathrm{O}\right) .{ }^{13} \mathrm{C}$ NMR $(75$ 
MHz, DMSO- $\left.d_{6}, \delta\right): 22.0\left(\mathrm{CH}_{3}\right), 47.2\left(\mathrm{CH}_{2}\right), 98.5\left(\mathrm{C}_{3}\right), 119.3,122.1,125.3,125.7,133.8,134.1$, 145.1, 147.6, 152.3, 168.1, 175.4. M/z (relative intensity): 316 [ $\left.\mathrm{M}^{+}, 35\right], 308$ (34), 249 (34), 224 (40), 199 (35), 192 (40), 186 (40), 177 (42), 153 (40), 149 (44), 135 (37), 128 (46), 118 (40), 116 (38), 111 (46), 101 (48), 85 (27) 67(37), 59(100). Anal. Calcd for $\mathrm{C}_{14} \mathrm{H}_{12} \mathrm{~N}_{4} \mathrm{O}_{5}$ (316.28): C, 53.17 ; H, 3.82; N, 17.71\%. Found: C, 53.04; H, 3.83; N, 17.54\%.

6-(1-Ethyl-4-hydroxy-2-oxo-1,2-dihydro-quinolin-3-yl)-5-nitro-4-oxo-3,4-dihydro-1H-

pyrimidin-2-ylidene-cyanamide (13). A mixture of compound 3 (3.02 g, $10 \mathrm{mmol})$ and cyanoguanide $(0.85 \mathrm{~g}, 10 \mathrm{mmol})$, in DMF $(50 \mathrm{~mL})$, was heated under reflux for $4 \mathrm{~h}$. The solid deposited after cooling was filtered and crystallized from dioxane to give compound $\mathbf{1 3}$ as yellow crystals, yield $(2.61 \mathrm{~g}, 71 \%)$, m.p. $268-270{ }^{\circ} \mathrm{C}$. IR $\left(\mathrm{KBr}, \mathrm{cm}^{-1}\right): 3435(\mathrm{OH}), 3190(\mathrm{NH})$, 3030 ( $\left.\mathrm{CH}_{\text {arom}}\right), 2978,2928$ ( $\left.\mathrm{CH}_{\text {aliphatic }}\right), 2199(\mathrm{CN}), 1646\left(\mathrm{C}=\mathrm{O}_{\text {quinolinone }}\right), 1616(\mathrm{C}=\mathrm{N}), 1586$ $(\mathrm{C}=\mathrm{C}) .{ }^{1} \mathrm{H}$ NMR $\left(300 \mathrm{MHz}, \mathrm{DMSO}-d_{6}, \delta\right): 1.23\left(\mathrm{t}, 3 \mathrm{H}, J=7.0 \mathrm{~Hz}, \mathrm{CH}_{2} \mathrm{CH}_{3}\right), 4.33(\mathrm{q}, 2 \mathrm{H}, J=$ $\left.7.0 \mathrm{~Hz}, \mathrm{CH}_{2} \mathrm{CH}_{3}\right), 7.33$ (t, $\left.1 \mathrm{H}, J=7.2 \mathrm{~Hz}, \mathrm{H}-6\right), 7.59$ (d, $\left.1 \mathrm{H}, J=8.0 \mathrm{~Hz}, \mathrm{H}-8\right), 7.94(\mathrm{t}, 1 \mathrm{H}, J=$ $7.2 \mathrm{~Hz}, \mathrm{H}-7), 8.07$ (d, 1H, J=8.0 Hz, H-5), 12.62, $13.40\left(2 \mathrm{~s}, 2 \mathrm{H}, 2 \mathrm{NH}\right.$ exchangeable with $\left.\mathrm{D}_{2} \mathrm{O}\right)$, 13.95 (s, $1 \mathrm{H}, \mathrm{OH}$ exchangeable with $\left.\mathrm{D}_{2} \mathrm{O}\right) . \mathrm{M} / \mathrm{z}$ (relative intensity): 368 [ $\left.\mathrm{M}^{+}, 90\right], 367$ [11], 355(37), 337 (38), 322 (43), 298 (45), 297 (53), 293 (48), 273 (46), 232 (40), 213 (29), 205 (31), 147 (49), 131 (61), 118 (40), 103 (49), 77(43), 69(100). ${ }^{13} \mathrm{C}$ NMR (75 MHz, DMSO- $\left.d_{6}, \delta\right): 12.8$ $\left(\mathrm{CH}_{3}\right), 37.4\left(\mathrm{CH}_{2}\right), 103.8\left(\mathrm{C}_{3}\right), 116.0,118.7,122.5,123.4,128.1,131.6,136.5,138.5,140.4$, 156.7, 157.4, 166.6, 184.6. Anal. Calcd for $\mathrm{C}_{16} \mathrm{H}_{12} \mathrm{~N}_{6} \mathrm{O}_{5}$ (368.31): C, 52.18; H, 3.28; N, 22.82\%. Found: C, 52.06; H, 3.18; N, 22.27\%.

1-Ethyl-4-hydroxy-3-(2-methylsulfanyl-5-nitro-6-oxo-3,6-dihydro-pyrimidin-4-yl)-quinolin2(1H)-one (14). A mixture of compound 3 (3.20 g, $10 \mathrm{mmol})$ and S-methylisothiourea (0.09 g, $10 \mathrm{mmol}$ ), in DMF (30 mL), was heated under reflux for $4 \mathrm{~h}$. The yellow crystals obtained after cooling was filtered and recrystallized from $\mathrm{DMF} / \mathrm{EtOH}$ to give compound $\mathbf{1 4}$ as yellow crystals, yield $\left(2.46\right.$ g, 66\%), m.p. $244-246{ }^{\circ} \mathrm{C}$. IR $\left(\mathrm{KBr}, \mathrm{cm}^{-1}\right): 3366,3285,3100(\mathrm{OH}, \mathrm{NH}), 2971$ $\left(\mathrm{CH}_{\text {aliphatic }}\right), 1655\left(\mathrm{C}=\mathrm{O}_{\text {quinolinone }}\right.$ and $\left.\mathrm{C}=\mathrm{O}_{\text {pyrimidine }}\right), 1605,1588,1570(\mathrm{C}=\mathrm{N}$ and $\mathrm{C}=\mathrm{C}) .{ }^{1} \mathrm{H}$ NMR $\left(500 \mathrm{MHz}, \mathrm{CDCl}_{3}, \delta\right): 1.34$ (t, 3H, J=6.6 Hz, $\left.\mathrm{CH}_{2} \mathrm{CH}_{3}\right), 2.83$ (t, 3H, $\left.\mathrm{SCH}_{3}\right), 4.36$ (q, $2 \mathrm{H}, J=6.6$ $\left.\mathrm{Hz}, \mathrm{CH}_{2} \mathrm{CH}_{3}\right), 7.32(\mathrm{t}, 1 \mathrm{H}, J=7.2 \mathrm{~Hz}, \mathrm{H}-6), 7.55-7.82(\mathrm{~m}, 2 \mathrm{H}, \mathrm{H}-8$ and $\mathrm{H}-7), 8.19(\mathrm{~d}, 1 \mathrm{H}, J=8.0$ $\mathrm{Hz}, \mathrm{H}-5), 13.14$ (s, 1H, NH exchangeable with $\mathrm{D}_{2} \mathrm{O}$ ), 13.39 (s, H, OH exchangeable with $\mathrm{D}_{2} \mathrm{O}$ ). ${ }^{13} \mathrm{C}$ NMR (125 MHz, DMSO-d $\left.d_{6}, \delta\right): 12.6\left(\mathrm{CH}_{3}\right), 22.1\left(\mathrm{SCH}_{3}\right), 43.7\left(\mathrm{CH}_{2}\right), 101.2,111.8,114.7$, $116.0,122.2,123.5,124.2,133.9,137.3,138.7,154.7,157.3,158.3 . \mathrm{M} / \mathrm{z}$ (relative intensity): 375 $\left[\mathrm{M}^{+}+1,37\right], 374$ [M $\left.\mathrm{M}^{+}, 100\right], 326$ (20), 311 (61), 297 (17), 195 (35), 280 (16), 252 (13), 237 (4), 194 (12), 150 (24), 136 (25), 111 (35), 94 (23), 59 (14). Anal. Calcd for $\mathrm{C}_{16} \mathrm{H}_{14} \mathrm{~N}_{4} \mathrm{O}_{5} \mathrm{~S}$ (374.38): C, 51.33; H, 3.77; N, 14.97; S, 8.56\%. Found: C, 51.12; H, 3.63; N, 14.74, S, 8.36\%.

\section{1-Ethyl-4-hydroxy-3-(2-hydrazino-5-nitro-6-oxo-3,6-dihydro-pyrimidin-4-yl)-quinolin-}

2(1H)-one (15). A mixture of compound 14 (3.74 g, $10 \mathrm{mmol})$, and hydrazine hydrate $(0.58 \mathrm{~mL}$, $12 \mathrm{mmol}$ ), in DMF (30 mL), was refluxed for $2 \mathrm{~h}$. The precipitate so formed on hot was filtered and crystallized from acetic acid, to give compound $\mathbf{1 5}$ as coulorless crystals, yield (2.68 g, 75\%), m.p. 322-324 ${ }^{\circ} \mathrm{C}$. IR $\left(\mathrm{KBr}, \mathrm{cm}^{-1}\right): 3411,3335,3191,3100\left(\mathrm{OH}, \mathrm{NH}_{2}, \mathrm{NH}\right), 3070$ $\left(\mathrm{CH}_{\text {arom. }}\right), 2979,2937$ ( $\left.\mathrm{CH}_{\text {aliphatic }}\right), 1654\left(\mathrm{C}=\mathrm{O}_{\text {quinoline }}\right.$ and $\left.\mathrm{C}=\mathrm{O}_{\text {pyrimidine }}\right), 1600,1567(\mathrm{C}=\mathrm{N}$ and 
$\mathrm{C}=\mathrm{C}) .{ }^{1} \mathrm{H}$ NMR (500 MHz,DMSO- $\left.d_{6}, \delta\right): 1.25\left(\mathrm{t}, 3 \mathrm{H}, J=6.4 \mathrm{~Hz}, \mathrm{CH}_{2} \mathrm{CH}_{3}\right), 4.37$ (q, $2 \mathrm{H}, J=6.6$ $\mathrm{Hz}, \mathrm{CH}_{2} \mathrm{CH}_{3}$ ), 7.16 (br, $2 \mathrm{H}, \mathrm{NH}_{2}$ exchangeable with $\mathrm{D}_{2} \mathrm{O}$ ), 7.50 (t, $\left.1 \mathrm{H}, J=7.2 \mathrm{~Hz}, \mathrm{H}-6\right), 7.85-$ $7.90(\mathrm{~m}, 2 \mathrm{H}, \mathrm{H}-8$ and $\mathrm{H}-7), 8.13(\mathrm{~d}, 1 \mathrm{H}, J=8.0 \mathrm{~Hz}, \mathrm{H}-5), 8.25,11.48(2 \mathrm{~s}, 2 \mathrm{H}, 2 \mathrm{NH}$ exchangeable with $\left.\mathrm{D}_{2} \mathrm{O}\right), 14.56\left(\mathrm{~s}, 1 \mathrm{H}, \mathrm{OH}\right.$ exchangeable with $\left.\mathrm{D}_{2} \mathrm{O}\right) . \mathrm{M} / \mathrm{z}$ (relative intensity):

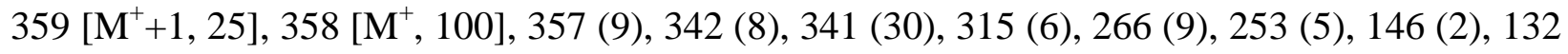
(7), 128 (10), 118 (15), 92 (8), 91 (39), 78 (10), 77 (86), 65 (14). Anal. Calcd for $\mathrm{C}_{15} \mathrm{H}_{14} \mathrm{~N}_{6} \mathrm{O}_{5}$ (358.32): C, 50.28; H, 3.94; N, 23.45\%. Found: C, 50.24; H, 3.83; N, $23.34 \%$.

\section{1-Ethyl-4-hydroxy-3-(1,5-dihydro-6-nitro-5-oxo-[1,2,4]triazolo[4,3-a]pyrimidin-7-yl)-} quinolin-2(1H)-one (16). To a solution of compound $15(3.58 \mathrm{~g}, 10 \mathrm{mmol})$ in DMF (30 mL), triethyl orthoformate $(2.04 \mathrm{~mL}, 12 \mathrm{mmol})$ was added and heated under reflux for $2 \mathrm{~h}$. The precipitate so formed on hot was filtered and crystallized from acetic acid, to give compound $\mathbf{1}$ as coulorless crystals, yield $(2.55 \mathrm{~g}, 69 \%)$, m.p. above $325^{\circ} \mathrm{C}$. IR $\left(\mathrm{KBr}, \mathrm{cm}^{-1}\right): 3400(\mathrm{OH}), 3292$, $3190(\mathrm{NH}), 3078\left(\mathrm{CH}_{\text {arom }}\right), 2970,2938\left(\mathrm{CH}_{\text {aliphatic }}\right), 1668\left(\mathrm{C}=\mathrm{O}_{\text {pyrimidine }}\right), 1632\left(\mathrm{C}=\mathrm{O}_{\text {quinoline }}\right)$ 1610, $1577(\mathrm{C}=\mathrm{N}$ and $\mathrm{C}=\mathrm{C}) .{ }^{1} \mathrm{H}$ NMR $\left(300 \mathrm{MHz}, \mathrm{DMSO}-d_{6}, \delta\right): 1.24(\mathrm{t}, 3 \mathrm{H}, J=6.6 \mathrm{~Hz}$, $\left.\mathrm{CH}_{2} \mathrm{CH}_{3}\right), 4.38\left(\mathrm{q}, 2 \mathrm{H}, J=6.6 \mathrm{~Hz}, \mathrm{CH}_{2} \mathrm{CH}_{3}\right), 7.55(\mathrm{t}, 1 \mathrm{H}, J=7.2 \mathrm{~Hz}, \mathrm{H}-6), 7.79-7.98(\mathrm{~m}, 2 \mathrm{H}, \mathrm{H}-$ 8 and $\mathrm{H}-7), 8.13(\mathrm{~d}, 1 \mathrm{H}, J=8.0 \mathrm{~Hz}, \mathrm{H}-5), 8.22\left(\mathrm{~s}, 1 \mathrm{H}, \mathrm{CH}_{\text {triazolopyrimidin }}\right), 11.33(\mathrm{~s}, 1 \mathrm{H}, \mathrm{NH}$ exchangeable with $\left.\mathrm{D}_{2} \mathrm{O}\right), 14.50\left(\mathrm{~s}, 1 \mathrm{H}, \mathrm{OH}\right.$ exchangeable with $\left.\mathrm{D}_{2} \mathrm{O}\right) . \mathrm{M} / \mathrm{z}$ (relative intensity): $369\left[\mathrm{M}^{+}+1,26\right], 368$ [M+, 100], 367 (57), 340 (50), 324 (24), 313 (80), 296 (21), 285 (15), 258 (6), 257 (12), 220 (10), 171 (11), 163 (10), 146 (40), 133 (66), 130 (28), 119 (44), 104 (30), 77(75), 63(75). Anal. Calcd for $\mathrm{C}_{16} \mathrm{H}_{12} \mathrm{~N}_{6} \mathrm{O}_{5}$ (368.31): C, 52.18; H, 3.28; N, 22.82\%. Found: $\mathrm{C}$, $52.15 ; \mathrm{H}, 3.58 ; \mathrm{N}, 23.54 \%$.

\section{References}

1. Tedesco, R.; Chai, D.; Darcy, M. G.; Dhanak, D.; Fitch, D. M.; Gates, A.; Johnston, V. K.; Keenan, R. M.; Lin-Goerke, J.; Sarisky, R. T.; Shaw, A. N.; Valko, K. L.; Wiggall, K. J.; Zimmerman M. N.; Duffy, K. J. Bioorg. Med. Chem. Lett. 2009, 19, 4354.

2. Hutchinson, D. K.; Flentge, C. A.; Donner, P. L.; Wagner, R.; Maring, C. J.; Kati, W. M.; Liu, Y.; Masse, S. V.; Middleton, T.; Mo, H.; Montgomery, D.; Jiang, W. W.; Koev, G.; Beno, D. W. A.; Stewart, K. D.; Stoll, V. S.; Molla, A.; Kempf, D. J. Bioorg. Med. Chem. Lett. 2011, 21, 1876.

3. Shiro, T.; Takahashi, H.; Kakiguchi, K.; Inoue, Y.; Masuda, K.; Nagata, H.; Tobe, M. Bioorg. Med. Chem. Lett. 2012, 22, 285.

4. Durón, S. G.; Lindstrom, A.; Bonnefous, C.; Zhang, Hui.; Chen, Xiaohong.; Symons, K. T.; Sablad, M.; Rozenkrants N.; Zhang Y.; Wang, Li.; Yazdani, Nahid.; Shiau, A. K.; Noble, S. A.; Rix, P.; Rao, T. S.; Hassig, C. A.; Smith, N. D. Bioorg. Med. Chem. Lett. 2012, 22, 1237.

5. Ghorab, M. M.; Heiba, H. I.; Amin, N. E. Pharmazie 1999, 54, 226. 
6. Mariah, F. E. L. J. Chem. Res. 2009, 9, 588.

7. Duraipandiyan, V.; Ignacimuthu, S. J. Ethnopharm. 2009, 123, 494.

8. El-Taweel, F. M. A.; Sofan, M. A.; Mashaly, M. A.; Hanna, M. A.; Elagamey, A. A. Pharmazie 1990, 45, 671.

9. Tavares, L. C.; Johann, S.; Alves, T. M. A.; Guerra, J. C.; de Souza-Fagundes, E. M.; Cisalpino, P. Silva.; Bortoluzzi, A. J.; Caramori, G. F.; Piccoli. R. M.; Braibante, H. T. S.; Braibante, M. E. F.; Pizzolatti, M. G. Eur. J. Med. Chem. 2011, 46, 4448.

10. Kalkhambkar, R. G.; Aridoss, G.; Kulkarni, G. M.; Bapset, R. M.; Mudaraddi, T. Y.; Premkumar, N.; Jeong, Y. T. Monatsh Chem. 2011, 142, 305.

11. Magedov, I. V.; Manpadi, M.; Ogasawara, M. A.; Dhawan, A. S.; Rogelj, S.; Van Slambrouck, S.; Steelant, W. F. A.; Evdokimov, N. M.; Uglinskii, P. Y,; Elias, E. M.; Knee, E. J.; Tongwa, P.; Antipin, Y. M.; Kornienko, A. J. Med. Chem. 2008, 51, 2561.

12. Guo, R.-H; Zhang, Q.; Ma, Y.-B.; Huang, X.-Y; Luo, J.; Wang, L.-J; Geng, C.-A; Zhang, X.-M; Zhou, J.; Jiang, Z.-Y; Chen, J.-J. Bioorg. Med. Chem. 2011, 19, 1400.

13. Guo, R.-H.; Zhang, Q.; Ma,Y.-B.; Luo, J. Geng, C.-A.; Wang, L.-J.; Zhang, X.-M.; Zhou, J.; Jiang, Z.-Y.; Chen. J.-J. Eur. J. Med. Chem. 2011, 46, 307.

14. Curreli, F.; Zhang, H.; Zhang, X.; Pyatkin, I.; Victor, Z.; Altieri, A.; Debnath, A. K. Bioorg. Med. Chem. 2011, 19, 77.

15. Abass, M.; Othman, E. S.; Hassan, A. Synth. Commun. 2007, 37, 607.

16. Abass, M; Mostata, B. B. Bioorg. Med. Chem. 2005, 13, 6133.

17. Roschger, P.; Fiala, W.; Stadlbauer, W. J. Heterocyclic Chem. 1992, 29, 225.

18. Tabacović, K.; Tabacović, I.; Trkovnik, M.; Trinajstić, N. Liebigs Ann. Chem. 1983, 1901.

19. Jung, J-C.; Oh, S.; Kim, W-K.; Park, W-K.; Kong, J. Y.; Park, O-S. J. Heterocyclic Chem., 2003, 40, 617.

20. López Rivilli, M. J.; Moyano, E. L.; Yranzo, G. I. Tetrahedron Lett. 2010, 51, 478.

21. Christodoulou, M. S.; Liekens, S.; Kasiotis, K. M.; Haroutounian, S. A. Bioorg. Med. Chem. 2010, 18, 4338.

22. Pawlas, J.; Vedso, P.; Jakobsen, P.; Huusfeldt, P. O.; Begtrup, M. J. Org. Chem. 2000, 65, 9001.

23. Abonía, R.; Cuervo, P.; Hursthouse, M. B.; Cobo, J.; Glidewell, C. Acta Cryst. Sect. C: Crystal Str. Commun. 2010, 66, 44.

24. Gao, W.-T.; Hou, W.-D.; Zheng, M.-R.; Tang, L.-J. Synth. Commun. 2010, 40, 732.

25. El-Taweel, F. M. A.; Sowellim, S. Z. A.; Elagamey, A. G. Boll. Chim. Farmac. 1998, 137, 325.

26. Stadlbauer, W.; Prattes, S.; Fiala, W. J. Heterocyclic Chem. 1998, 35, 627.

27. Hojas, G.; Fiala, W.; Stadlbauer, W. J. Heterocyclic Chem. 2000, 37, 1559.

28. Ovchinnikov, I. V.; Strelenko, Yu. A.; Popov, N. A.; Finogenov, A. O.; Makhova, N. N.; Russ. Chem. Bull., Int. Ed., 2011, 60 (5), 855.

29. (a) Hassner, A.; Stumer, C. Tetrahedron Organic Chemistry Series Vol. 11: Organic Syntheses Based on Name Reactions and Unnamed Reactions (Baldwin, J. E.; Magnus, P. 
D., Eds.), Pergamon, Oxford, 1994. (b) Krauch, H.; Kunz, W.; Nonnenmacher, E.; Reaktionen der Chemie, Dr, Verlag, A. H. Heidelberg, 1976.

30. Ahvale, A. B.; Prokopcová, H.; Šefčovičová, J.; Steinschifter, W.; Täubl, A. E.; Uray, G.; Stadlbauer, W. Eur. J. Org. Chem. 2008, 563. 